\title{
Automatic Hand Measurement System from 2D Hand Image for Customized Glove Production
}

\author{
Hyun Sook Han ${ }^{1)}$ and Chang Kyu Park ${ }^{2) \dagger}$ \\ ${ }^{1)}$ The research institute of Computer Information and Communication, Korea University, Seoul, Korea \\ ${ }^{2)}$ Department of Organic and Nano System Engineering, Konkuk University, Seoul, Korea
}

\begin{abstract}
Recent advancements in optics technology enable us to realize fast scans of hands using two-dimensional (2D) image scanners. In this paper, we propose an automatic hand measurement system using 2D image scanners for customized glove production. To develop the automatic hand measurement system, firstly hand scanning devices has been constructed. The devices are designed to block external lights and have user interface to guide hand posture during scanning. After hands are scanned, hand contour is extracted using binary image processing, noise elimination and outline tracing. And then, 19 hand landmarks are automatically detected using an automatic hand landmark detection algorithm based on geometric feature analysis. Then, automatic hand measurement program is executed based on the automatically extracted landmarks and measurement algorithms. The automatic hand measurement algorithms have been developed for 18 hand measurements required for custom-made glove pattern making. The program has been coded using the $\mathrm{C}++$ programming language. We have implemented experiments to demonstrate the validity of the system using 11 subjects (8 males, 3 females) by comparing automatic 2D scan measurements with manual measurements. The result shows that the automatic 2D scan measurements are acceptable in the customized glove making industry. Our evaluation results confirm its effectiveness and robustness.
\end{abstract}

Key words : hand measurement, custom-made gloves, automatic measurement, 2D hand scanning

\section{Introduction}

Importance of gloves is getting higher in sports (golf and baseball) and in surgery. Moreover, to be able to efficiently use hands fitted with gloves, it is extremely important to wear gloves of appropriate sizes and shapes. In particular, well-fitted gloves enhance performances and enable effective and precise hand movements. Lastly, the 1988 US Army hand anthropometric survey report (Greiner, 1991; Hidson, 1991) provided statistical information of 86 hand dimensions (1003 males and 1304 females). Glove companies employing mass production techniques use their own glove sizing systems to provide better fits for customers having different hand sizes (Kwon et al, 2009; Rosenblad-Wallin, 1987; Ryu \& Suh, 2004).

However, human hands have widely varying lengths, widths, and depths (Choi \& Kim, 2004; Linghua et al., 2009), and it is difficult for mass production systems to provide the best fit for all

†Corresponding author; Chang Kyu Park

Tel. +82-2-450-4196, Fax. +82-2-452-4196

E-mail: cezar@konkuk.ac.kr

(C) 2016 (by) the authors. This article is an open access article distributed under the terms and conditions of the Creative Commons Attribution license (http://creativecommons.org/licenses/by/3.0/), which permits unrestricted use, distribution, and reproduction in any medium, provided the original work is properly cited. types of hands. Therefore, custom-made gloves can give the best fit to individuals. However, until now, making custom-made gloves has been time consuming and has involved direct contact with human subjects since the hands had to be measured manually.

Today, advancements in optics technology enable us to obtain fast scans by using three-dimensional (3D) hand scanners. The 3D scan method can measure all the dimensions that are measured by the manual method, such as, for example, the circumference and depth. However, it is difficult for glove companies to adopt this system because the 3D scanners are expensive and several limitations in scanning hands (Lee et al., 2010). An alternative method for measuring hand dimensions is based on the image analyses of $2 \mathrm{D}$ scan images. The cost of a $2 \mathrm{D}$ scanner is rather low, and its ease of usage is considerably better than that of the $3 \mathrm{D}$ hand scanner. However, the number of body dimensions that can be collected is relatively limited, and the approximated circumferences may be less accurate. Therefore, there is a tradeoff between the usages of 3D scanning data and 2D images for hand measurements.

A few researches have been conducted previously on 3D hand measurements. In one research, the 3D hand dimension extraction was validated ( $\mathrm{Li}$ et al., 2008), while in other researches, the 3D hand measurement protocol was studied (Chang et al., 2007; Lee et al., 2010). However, to our knowledge, investigations into automatic hand measurements using $2 \mathrm{D}$ image analyses are few. 
In this study, we have developed an automatic hand measurement system based on the image analysis of 2D scan images. In this paper, in Section 2, we describe the extraction of a hand contour from the hand scan image. Section 3 presents automatic hand landmark detection algorithms. In Section 4, automatic hand measurement procedures using the hand landmarks are described. Subsequently, in Section 5, experiments to validate the system have been described. Finally, their results-with emphasis on the important findings-are described in Section 6, along with the major conclusions of the study.

\section{Hand Scan Data Acquisition}

\subsection{Hand scanning}

A 2D image scanner was used to acquire hand image data. The 2D scanner was CanoScan LiDE 110 and its resolution was $2400 \times 4800$. To extract the hand contour, it is necessary to contrast the brightness of the hand and background. Hence, we blocked the external light by enclosing the scanner within a self-produced case and making the background color black (Fig. 1(a)). We scanned psalm side of the hand because the psalm is more flat and hand silhouette could be scanned more exactly.

A user interface to guide the hand scanning was developed. Fig. 1(b) shows the interface. We determined name of the system managing from 2D scanning to automatic hand measurement as Glove Customizer 2.1(Fig. 1). Before the scanning, firstly, whether the hand is the right or left one must be chosen. Left hand was used for the study. Further, during the hand scanning, the subjects must be asked to separate each finger in accordance with the guidelines given in the system interface so that the hand contour can be extracted clearly. The guideline is shown in Fig. 1(b). The vertical red line at center is for placement of middle finger and the two $\mathrm{x}$ shape is for placement of distinguishing point of index finger and middle finger. In our coordinate system, the leftward direction is the $\mathrm{x}$-axis and the upward direction is the $\mathrm{y}$-axis..

\subsection{Extraction of hand contour}

Before the automatic hand landmark identification, the hand contour must be extracted from the hand scan image. The hand contour extraction procedure is as follows:

(1) Binary image processing: Calculate the average brightness of the R, G, B of all the pixels in the hand scan image. If the average brightness is smaller than 32 (dark), it is considered 0 , while, otherwise, it is 255 ; this leads to a white (hand) and black (black background) image. The result of binary image processing is shown in Fig. 2(b).

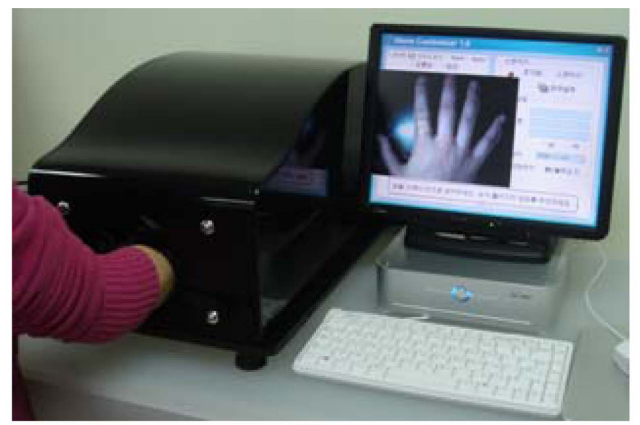

(a)

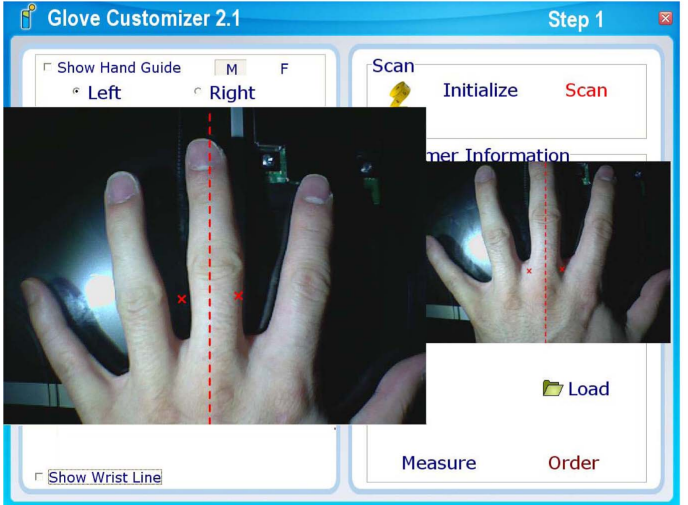

(b)

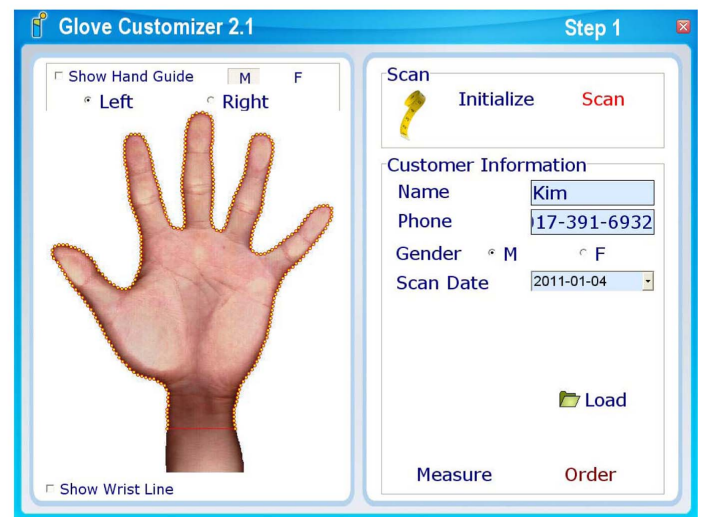

(c)

Fig. 1. Hand scanning system: (a) hand scan device, (b) scanning process, (c) hand contour extraction. 


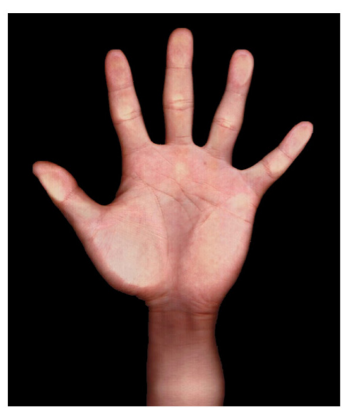

(a)

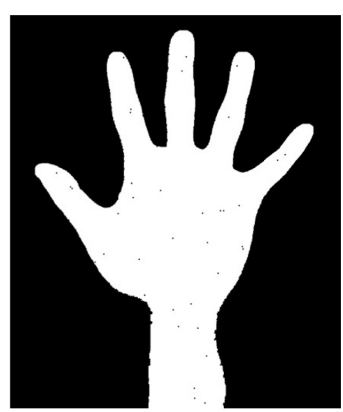

(b)

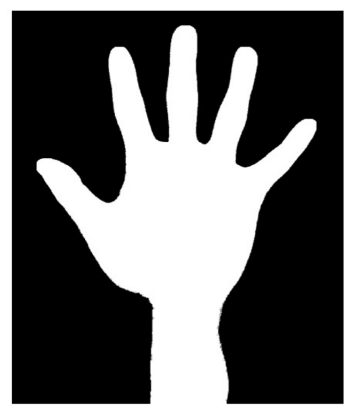

(c)

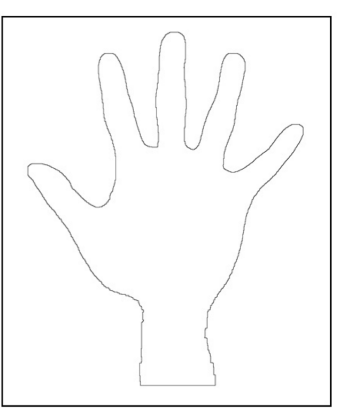

(d)

Fig. 2. Illustration of hand contour extraction: (a) color scan image, (b) binary image, (c) image after noise reduction, (d) hand contour extraction.

(2) Noise elimination: Eliminate small noises by employing two erosion processes followed by several dilation processes. The result of binary image processing is shown in Fig. 2(c).

(3) Hand contour extraction: Keep only the black pixels with at least one neighboring white pixel and erase all other pixels as white in the binary image. The result of binary image processing is shown in Fig. 2(d).

(4) Outline tracing: An arbitrary pixel on the hand contour is selected as the start pixel. Find the black pixel from among the eight pixels around the start pixel and perform the same operation with the black pixel recursively. Stop this operation when it returns to the start pixel.

(5) Convert to real coordinates in the following manner.

The origin is center of entire outline and the dimension is $\mathrm{mm}$. We know the scanner's dpi (dots per inch) and we can know the real coordinate from image. The real coordinates are calculated as follows:

$$
P_{x}=\frac{I_{x} \times 25.4}{\mathrm{dpi}}, P_{y}=\frac{I_{y} \times 25.4}{\mathrm{dpi}}
$$

$P_{x, y}=$ real coordinate, $I_{x, y}=$ image coordinate, dpi $=$ dot per inch of image

In above function, $I_{x, y}$ means image coordinate, position of each $\operatorname{dot}\left(=\right.$ pixel). $P_{x, y}$ can be calculated by dividing $I_{x, y}$ by dpi, dot per $\operatorname{inch}(=25.4 \mathrm{~mm})$ of image.

\subsection{Hand contour arrangement}

In order to search the hand landmarks more effectively, we rearranged the hand contour points. The lowest point at the radial side was considered the first point, and sequentially following the nearest points, the lowest point at the ulnar side became the final point.

\section{Automatic Hand Landmark Detection}

\subsection{Hand landmarks}

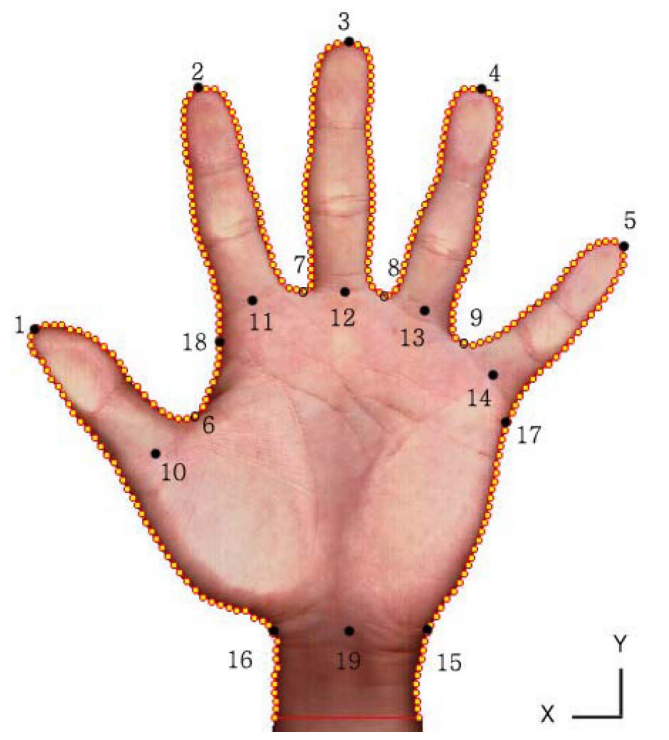

Fig. 3. Illustration of landmark locations.

In this study, we have automatically detected 19 hand landmarks. The definition of each landmark is listed in Table 1, while Fig. 3 illustrates the landmark locations on the hand.

\subsection{Automatic hand landmark detection methods}

After the hand contour tracing, the hand landmarks were detected automatically. The flowchart of the automatic hand landmark detection is given in Fig. 4. The detection order is determined respective of whether the landmark has to reference other landmarks. For example, the endpoint (radius side) of the middle wrist base line, P16, can be detected without other landmarks, and hence, it was detected first. Further, the previously detected hand landmarks are used for the follow-up detection of landmarks. The identification method of each landmark will be described in detail below and in Fig. 5. Here, the image of the left hand is used as the basis.

(1) Endpoint (radius side) of the middle wrist base line (P16) 
Table 1. Hand landmarks detected in this study

\begin{tabular}{lc}
\hline Notation & Hand landmarks \\
\hline P 1 & The tip of the thumb \\
P 2 & The tip of the index finger \\
P 3 & The tip of the middle finger \\
P 4 & The tip of the ring finger \\
P 5 & The tip of the little finger \\
P 6 & Distinguishing point between palm and thumb \\
P 7 & Distinguishing point between palm and index finger \\
P 8 & Distinguishing point between palm and middle finger \\
P 9 & Distinguishing point between palm and ring finger \\
P 10 & Midpoint of the middle crease line at palmar side of the metacarpalphalangeal joint of the thumb \\
P 11 & Midpoint of the middle crease line at palmar side of the metacarpalphalangeal joint line of the index finger (1st Crease Line, Thumb) \\
P 12 & Midpoint of the middle crease line at palmar side of the metacarpalphalangeal joint line of the middle finger \\
P 13 & Midpoint of the middle crease line at palmar side of the metacarpalphalangeal joint line of the ring finger \\
P 14 & Midpoint of the middle crease line at palmar side of the metacarpalphalangeal joint line of the little finger \\
P 15 & Endpoint (ulnar side) of the middle wrist base line \\
P 16 & Endpoint (radius side) of the middle wrist base line \\
P 17 & Metacarpale $V$ \\
P 18 & Metacarpale II \\
P 19 & Wrist center point
\end{tabular}

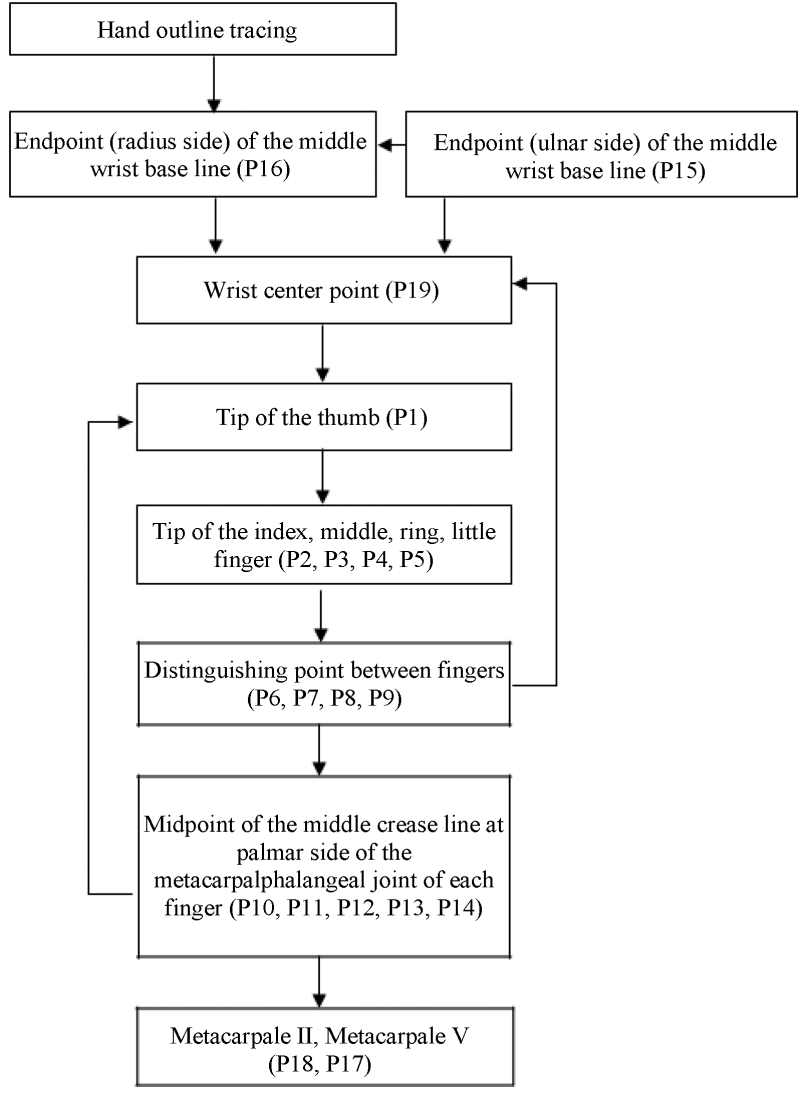

Fig. 4. Flowchart of hand landmark detection. (see Fig. 5a):

(a) Draw a tangent starting from the lowest right point $\left(\mathrm{P}_{\mathrm{Xmin}}\right)$ toward the thumb tip, and determine a tangent point ( $\left.\mathrm{P}_{\text {Tangent }}\right)$.

(b) Find the point of the largest distance from the line connecting $\mathrm{P}_{\mathrm{Xmin}}$ and $\mathrm{P}_{\text {Tangent. }}$ This point is determined to be the endpoint of the middle wrist base line.

(2) Endpoint (ulnar side) of the middle wrist base line (P15): Find a point at the same height as P16 and the largest $\mathrm{x}$ value.

(3) Wrist center (P19):

Find the middle position of P16 and P15.

(4) Tip of the thumb (P1) (see Fig. 5b):

(a) Examine the $y$ value starting from the first point of the hand contour and determine the point $\left(\mathrm{P}_{\text {Ydecrease }}\right)$ at which y begins to decrease.

(b) From among the outline points between the first point and $\mathrm{P}_{\text {Ydecrease, determine the point of the largest distance }}$ from the wrist center point (P19).

(5) Tip of the index, middle, ring, and little fingers (P2, P4, P5, and $\mathrm{P}$, respectively) (see Fig. 5c):

From among the outline points after P1, determine the point at which the slope changes from positive to negative and when the $y$ value of the next point is smaller 
than the current one.

(6) Distinguish the points between two fingers (P6, P7, P8, and P9):

From among the outline points between the left and right tip points, determine the point for the smallest distance from the wrist center point (P19).

(7) Midpoint of the middle crease line at the palmar side of the metacarpal-phalangeal joint of each point (P10, P11, P12, P13, and P14) (see Fig. 5d):

These five points are identified using the same method. Therefore, the identification method of P11 is presented as the representative method.

To find P11, first, the finger axis has to be set. The upper point of the axis is the finger tip point P2. The other bottom point was determined to be the midpoint of the horizontal line connecting two points at a height $20 \mathrm{~mm}$ above $\mathrm{P} 7$ from the points between $\mathrm{P} 7$ and $\mathrm{P} 6$. The finger axis line $\left(\mathrm{L}_{\text {Faxis }}\right)$ connects the bottom point and P2. Draw a line $\left(\mathrm{L}_{\text {bottom }}\right)$ passing through $\mathrm{P} 7$ and the perpendicular to the finger axis. The intersection between $\mathrm{L}_{\text {Faxis }}$ and $\mathrm{L}_{\text {bottom }}$ is $\mathrm{P} 11$.

(8) Metacarpal V (P17):

The metacarpal $\mathrm{V}$ bones are the bones of the hand, and they are located between the little fingers and wrist. The metacarpal $\mathrm{V}$ point is located at the upper exterior part of the metacarpal $\mathrm{V}$ bone. There are no prominent geometrical features, and it is hard to find the point using geometrical methods.

As an alternative method, we observe its relation with other landmarks and use this for identification. The metacarpal $\mathrm{V}$ is positioned below $\mathrm{P} 14$ at an average distance of $1.5 \mathrm{~cm}$ from the P14. Therefore, we set an outline point below $\mathrm{P} 14$ as metacarpal $\mathrm{V}$.

(9) Metacarpal II (P18)

Metacarpal II also has no prominent geometrical features, and we used its relation with other landmarks. Metarcarpal II is positioned at 4.5:5.5 between P6 and P11. Therefore, we set an outline point of this height as

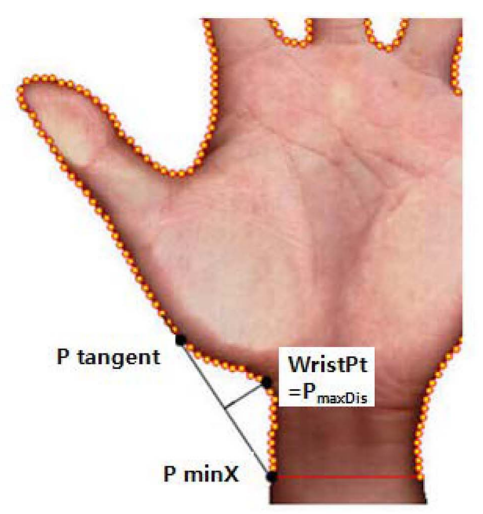

(a)

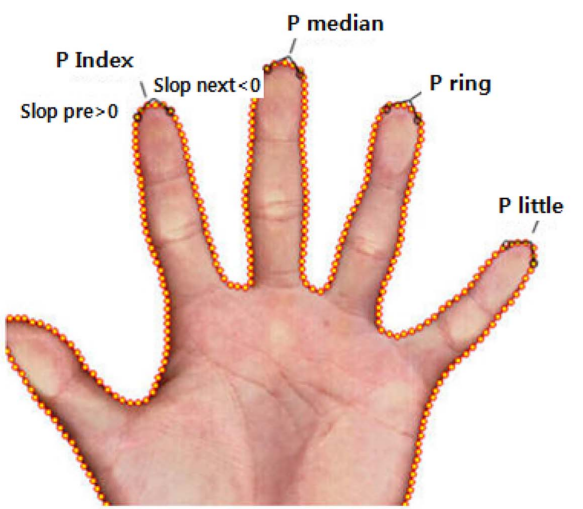

(c)

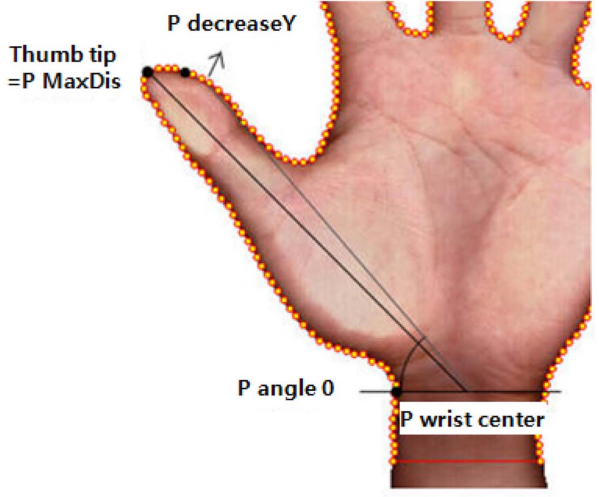

(b)

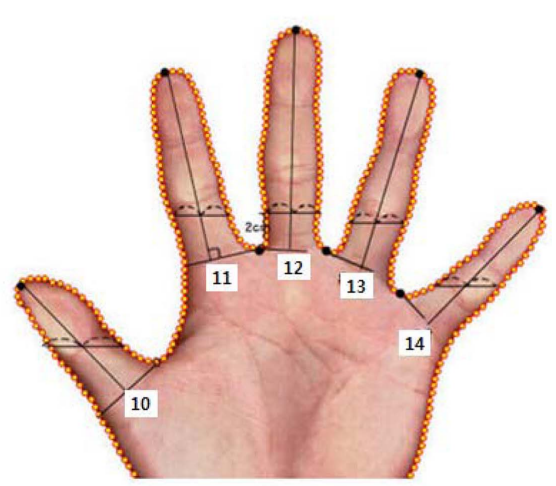

(d)

Fig. 5. Illustration of hand landmark detection method: (a) Endpoint (radius side) of the middle wrist base line, P16 (b) The tip of the thumb, P1 (c) The tip of the index, middle, ring, little finger, P2, P4, P5, P3 (d) Midpoint of the middle crease line at psalm side of the metacarpalphalangeal joint of each point (P10, P11, P12, P13, P14) 
metacarpal II (P18).

\section{Automatic Hand Measurements}

We measured 18 hand dimensions-comprising widths and lengths-that are required for custom-made glove pattern generations. Circumferences and depths could not be measured due to the limitations in 2D images. The dimensions are illustrated in Fig. 6. Most of these dimension definitions were referenced from ISO 7250-1 (International Organization for Standardization, 2008) and report on 'Development of hand measurement protocols for glove construction' (Ministry of Commerce, Industry and Energy, 2006).

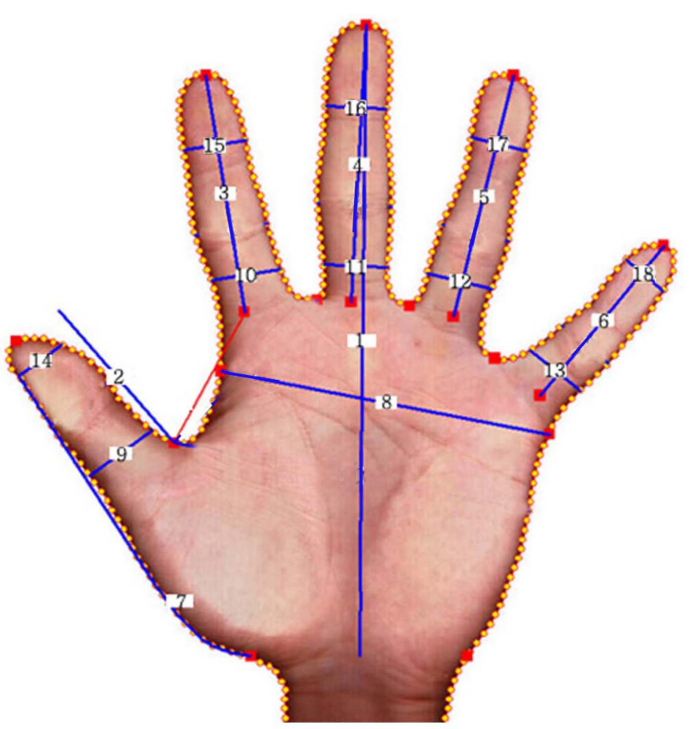

Fig. 6. Hand dimensions.

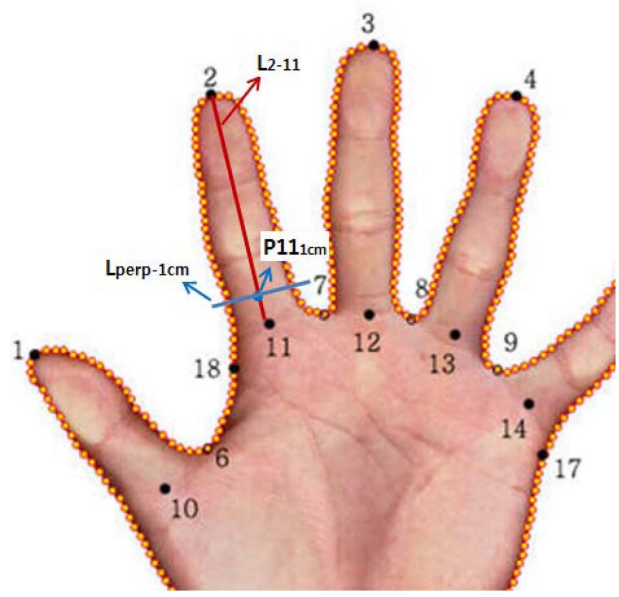

(a)
Table 2. Location estimates used to measure distal interphalangeal width

\begin{tabular}{ccccc}
\hline \multirow{2}{*}{ Item } & \multirow{2}{*}{ Finger } & \multicolumn{3}{c}{ Proportion (\%) } \\
\cline { 3 - 5 } & & Male & Female & Mean \\
\hline Ratio of sum of first & Thumb & 48.1 & 48.7 & 48.4 \\
phalanx & Index finger & 64.1 & 65.1 & 64.6 \\
\multirow{2}{*}{$\begin{array}{c}\text { length and second phalanx } \\
\text { length to finger length }\end{array}$} & Ring finger & 63.8 & 64.7 & 64.2 \\
& Little finger & 58.4 & 59.4 & 58.9 \\
\hline
\end{tabular}

\subsection{Automatic hand dimension measurement methods}

Linear dimensions comprising automatically detected landmarks can be easily obtained by simply calculating the distance between two landmarks. Therefore, in this section, we have described all dimensions other than linear ones.

(1) Metacarpal-phalangeal width (M9, M10, M11, M12, M13) (see Fig. 7a):

The measurement methods of the metacarpal-phalangeal width of five fingers are similar; in this study, the measurement method of M10 is presented as a representative.

(a) Find a point $\left(\mathrm{P} 11_{1 \mathrm{~cm}}\right) 1 \mathrm{~cm}$ above $\mathrm{P} 11$ on the index finger axis line $\mathrm{L}_{2-11}$ connecting $\mathrm{P} 11$ and $\mathrm{P}$.

(b) Make a line $\left(\mathrm{L}_{\text {perp-1 } \mathrm{cm}}\right)$ passing through the point $\mathrm{P} 11_{1 \mathrm{~cm}}$ and perpendicular to a line $\mathrm{L}_{2-11}$. Find two intersections between the line $\mathrm{L}_{\text {perp-1cm }}$ and hand contour points between P6 and P7. The distance between the two intersections is the metacarpal-phalangeal width of the index finger.

(2) Distal interphalangeal width (M15, M16, M17, M18) (see Fig. 7b):

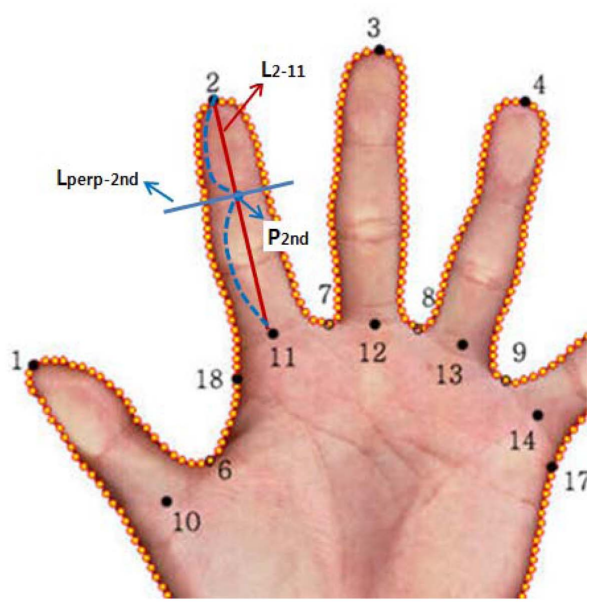

(b)

Fig. 7. Illustration of automatic hand dimension measurement methods: (a) Metacarpal-phalangeal width (M9, M10, M11, M12, M13, (b) Distal interphalangeal width (M15, M16, M17, M18). 
The location of the distal interphalangeal crease line cannot be identified using geometrical methods because there are no prominent geometrical features at the hand contour position. Therefore, statistical locations were used to determine the position of the distal interphalangeal width. The Korean hand anthropometry data (Korean Agency for Technology Standards, 2008) was used to calculate the position of the distal interphalangeal crease line. The proportional location of the distal interphalangeal crease line was calculated by dividing the sum of the first and second phalanx lengths by the finger length at each finger. The results are listed in Table 2 .

The measurement method of the distal interphalangeal width of the index finger is presented as a representative.

(a) Find a point $\left(\mathrm{P}_{2 n d}\right)$ that is $64.6 \%$ from $\mathrm{P} 2$ in the line $\left(\mathrm{L}_{2-11}\right)$ connecting $\mathrm{P} 11$ and $\mathrm{P} 2$. The value $64.6 \%$ represents the mean ratio of the sum of the first and second phalanx lengths to the finger length of the index finger.

(b) Draw a line $\left(\mathrm{L}_{\text {perp-2nd }}\right)$ passing through the point $\mathrm{P}_{2 \text { nd }}$ and perpendicular to a line $\mathrm{L}_{2-11}$. Find two intersections between the line $\mathrm{L}_{\text {perp-2nd }}$ and the hand contour points between P6 and P7. The distance between the two intersections is the distal interphalangeal width of the index finger.

\section{System Implementation and Validation}

The system, from 2D hand scan image processing to hand measurement result view, was coded using the $\mathrm{C}++$ programming language. Fig. 8 shows the "result view" of the interface of the developed hand measurement system. To validate the system, we scanned eleven subjects ( 8 males, 3 females; mean \pm S.D.; age $=38 \pm 7.2$ years; height $=169.6 \pm 6 \mathrm{~cm}$; body mass $=65.8 \pm 11.8 \mathrm{~kg}$ ) with no disorders in hand. They were randomly selected from the researchers of i-Fashion clothing technology center, Seoul, Korea. All subjects provided informed consent.

First, the subjects' left hand was scanned using the 2D hand

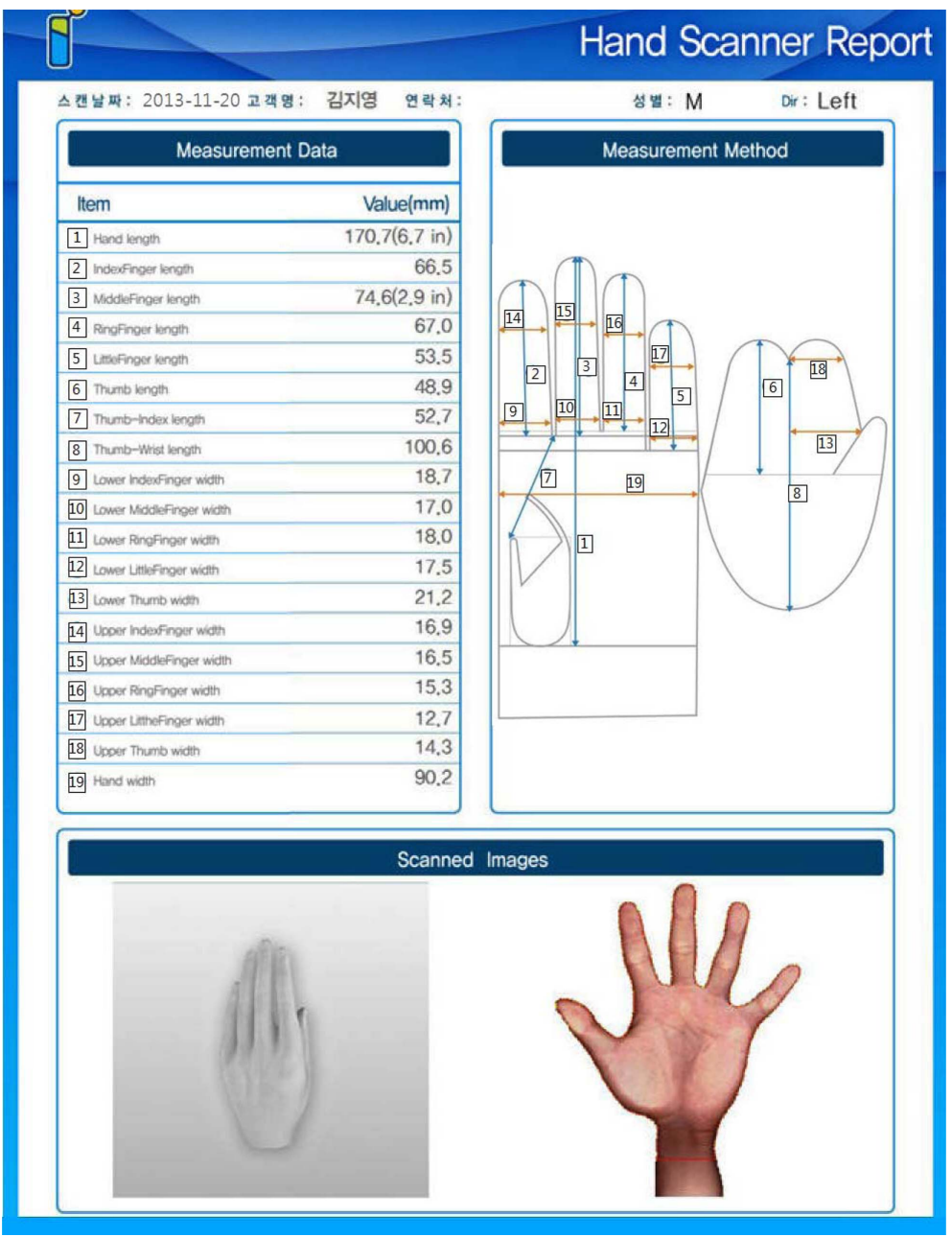

Fig. 8. Hand measurement result view of the system. 
scanner. Subsequently, manual measurements were conducted. The hand pose-each finger separated, psalm side of the hand up-was kept identical during both the manual and scan measurements by placing the hand on a printed paper of the scanned hand during the manual measurements. We measured 18 hand dimensions, the same dimensions measured in the automatic hand measurement system. Manual measurement method also followed definitions presented in ISO 7250-1 (International Organization for Standardization, 2008) and report on 'Development of hand measurement protocols for glove construction' (Ministry of Commerce, Industry and Energy, 2006), the same method of automatic hand measurement developed above. We marked each landmark with pen and measured the hand dimensions with calipers. After the scanning, the landmarks were automatically extracted, and the hand dimensions were measured by the developed system.

In order to evaluate the accuracy of the measurements obtained by the proposed automatic measurement method, all 18 hand dimensions were compared individually using the mean absolute difference (MAD) and the paired T-test. The results of the mean difference and t-tests are listed in Table 3. The paired T-tests indicated that significant differences were present between the values obtained by the two methods in the case of the hand length, index length, and (almost) finger breadth dimensions. The mean absolute difference(MAD) exceed the maximum allowable error-1 $\mathrm{mm}$ suggested by ISO 20685:2005-in several dimensions, hand length, most finger length and thumb width. However they are all within $2 \mathrm{~mm}$ except for hand length. The dimension item within $2 \mathrm{~mm}$ was regarded as marginal for glove making in this paper. This indicates that the measurements are acceptable in the glove making industry.

The large difference in the hand lengths(M1) can be explained by the poorly scanned wrist part. During the scanning, the wrist cannot be scanned completely but is scanned slightly inward; this is because the wrist has a thicker circular cross-section than the psalm and finger parts, and accordingly, the actual wrist outline is placed high above the scan plate making accurate scanning difficult. For the wrist outline scanned slightly inward, the concave point of the wrist is placed in more upper part than actual concave point and it decreases the hand length. Moreover, when the hand is rotated toward the ulna side, the wrist of the radius side is placed upward and the hand length decreases. In order to reduce the differences in the hand length, the wrist must be placed close to the scan plate and the hand must be rotated toward the thumb rather than the little finger.

The large difference in distal thumb breadth(M9) can be explained by the thumb posture. When a hand is placed on the plate, bottom of the thumb is not placed face down but it is rotated inward and the bottom of the thumb can not scanned completely. In order to reduce the differences in the thumb breadth, the thumb alone can be scanned separately.

The differences between the repeated scans did not exceed the

Table 3. Mean comparison between automatic and manual measurement methods $(n=11)$

(unit: $\mathrm{mm}$ )

\begin{tabular}{|c|c|c|c|c|c|c|c|c|c|c|}
\hline & Dimension & $\begin{array}{c}\text { Repeata } \\
\text { bility }\end{array}$ & $\begin{array}{c}\text { Manual } \\
\text { Mean }\end{array}$ & $\begin{array}{c}\text { Manual } \\
\text { S.D }\end{array}$ & $\begin{array}{l}\text { Scan } \\
\text { Mean }\end{array}$ & $\begin{array}{l}\text { Scan } \\
\text { S.D }\end{array}$ & $\begin{array}{c}\text { M.D } \\
\text { (scan-manual) }\end{array}$ & M.A.D & $\begin{array}{c}\text { S.D. } \\
\text { of } \mathrm{MD}\end{array}$ & $\begin{array}{c}\mathrm{t} \text { test } \\
p \text { value }\end{array}$ \\
\hline M 1 & Hand length & 0.72 & 183.1 & 9.0 & 176 & 9.0 & -7.0 & 7.4 & 3.1 & $<0.001$ \\
\hline M 2 & Thumb length & 0.52 & 51.4 & 2.4 & 51.4 & 2.4 & 0.0 & 1.4 & 1.7 & 0.456 \\
\hline M 3 & Index Finger length & 0.36 & 69.8 & 3.4 & 71.3 & 3.4 & 1.5 & 1.7 & 1.1 & $<0.01$ \\
\hline M 4 & Middle Finger length & 0.16 & 77.7 & 4.5 & 78.4 & 4.5 & 0.7 & 1.6 & 2.3 & 0.492 \\
\hline M 5 & Ring Finger length & 0.28 & 69.5 & 2.8 & 69.2 & 2.8 & -0.3 & 1.0 & 1.2 & 0.378 \\
\hline M 6 & Little Finger length & 0.52 & 55.2 & 3.5 & 55.4 & 3.5 & 0.2 & 1.3 & 1.8 & 0.798 \\
\hline M 8 & Hand breadth & 0.04 & 85.4 & 5.1 & 85.7 & 5.1 & 0.3 & 0.8 & 1.1 & 0.423 \\
\hline M 9 & Thumb breadth, bottom & 0.32 & 28.2 & 2.2 & 21.2 & 2.2 & 1.2 & 1.4 & 1.4 & $<0.05$ \\
\hline M 10 & Index Finger Breadth, bottom & 0.28 & 20.3 & 2.0 & 20.3 & 2.0 & 0.8 & 0.8 & 0.5 & $<0.001$ \\
\hline M 11 & Medius Finger Breadth, bottom & 0.24 & 17.7 & 2.0 & 19.2 & 2.0 & 0.6 & 0.8 & 0.8 & $<0.01$ \\
\hline M 12 & Ring Finger Breadth, bottom & 0.08 & 18.8 & 1.9 & 19.5 & 1.9 & 1.0 & 1.0 & 0.6 & $<0.001$ \\
\hline M 13 & Little Finger Breadth, bottom & 0.08 & 18.1 & 2.0 & 17.8 & 2.0 & 1.0 & 1.0 & 0.6 & $<0.001$ \\
\hline M 14 & Thumb breadth, distal & 0.12 & 22.3 & 2.6 & 15.3 & 2.6 & -2.9 & 3.1 & 2.4 & $<0.001$ \\
\hline M 15 & Index Finger Breadth, distal & 0.08 & 17.3 & 1.2 & 17.3 & 1.2 & 0.7 & 0.8 & 0.6 & $<0.01$ \\
\hline M 16 & Medius Finger Breadth, distal & 0.08 & 15.8 & 1.3 & 17.3 & 1.3 & 0.7 & 0.8 & 0.4 & $<0.001$ \\
\hline M 17 & Ring Finger Breadth, distal & 0.08 & 15.6 & 1.3 & 16.3 & 1.3 & 0.8 & 0.8 & 0.5 & $<0.001$ \\
\hline M 18 & Little Finger Breadth, distal & 0.08 & 14.8 & 1.5 & 14.5 & 1.5 & 0.5 & 0.6 & 0.7 & 0.034 \\
\hline
\end{tabular}


acceptable threshold, $1 \mathrm{~mm}$ for scanner-derived measurements (International Organization for Standardization, 2005) in all measurements. Table 3 shows the repeatability, mean difference of 5 repeat measurement of one subject. This shows that the system generates reliable measurements.

This study suggested regression equation for three measurements showed comparatively large error, hand length(M1), thumb breadth bottom(M9) and thumb breadth distal(M14) to translate the scan measurement to more accurate value. The regression analysis was conducted with the same data used for system validation 11 subjects. In the analysis, manual measurement was set as dependant variable and scan measurement as independent variable. The equation of each measurement is as follows;

- Hand length(M1) $=0.9105 \times$ scan hand length +23.12

- Thumb breadth bottom(M9) $=1.33 \times$ scan thumb breadth bottom -8.23

- Thumb breadth distal(M1) $=1.59 \times$ scan thumb breadth distal $-6.08$

\section{Conclusion}

In this paper, an automatic hand measurement system for customized glove making by using 2D image scanners has been described. The system involves hand contour extraction, automatic hand landmark detections, and automatic hand measurements. For automated landmarking, hand contour extraction was performed, and the identification order was determined. Subsequently, detection algorithms were proposed for 19 landmarks. Eighteen hand dimensions were then obtained automatically. To evaluate the validity of the newly developed system, 11 subjects' left hand was scanned and tested. Our evaluation results suggest that the system was effective and robust in most dimensions except for hand length and thumb breadth. To reduce the differences in the hand length and thumb breadth, a method to scan the wrist and thumb accurately have to be studied further. Also, evaluation has to be done with more many numbers of subjects and with diverse hand types, for example fat and thin hand.

Even if our measurement method proves to be able to estimate hand anthropometric measurements satisfactorily, the link from good hand data to a successful "customized glove making" outcome is a completely different test. Also, this study has limitation that it can't measure depth dimension because it uses only psalm side 2D image of the hand. Therefore, further study on converting 2D information to 3D products including depth dimension has to be done also. However, we believe that the system helpful in making a good fitness of customized gloves and reduces human efforts, costs, and production time.

\section{Acknowledgements}

This paper was written as part of Konkuk University's research support program for its faculty on sabbatical leave in 2016.

\section{References}

Chang, C. C., Li, Z. Z., Cai, X. W., \& Dempsey, P. (2007). Error control and calibration in three-dimensional anthropometric measurement of the hand by laser scanning with glass support. Measurement, 40(1), 21-27.

Choi, H. S., \& Kim, E. K. (2004). The database development of 2-D and 3-D hands measurement for improving fitness of glovesfocused on the classification of hand type and analysis of 3-D hand shape. Journal of the Korean Society of Clothing and Textiles, 28(9), 1300-1311.

Greiner, T. M. (1991). Hand anthropometry of U.S. Army Personnel. U.S. Army Natick research, Development and Engineering Center. Natick: MA. (NTIS No.ADA244533).

Hidson, D. (1991). Development of a standard anthropometric dimension set for use in computer-aided glove design. DREO Technical Note 91-22, Defense Research Establishment OTTAWA.

International Organization for Standardization. (2008). ISO 7250-1: Basic human body measurements for technological design-Part 1: Body measurement definitions and landmarks.

Korean Agency for Technology Standards. (2008). Final report on the Korean hand anthropometry data.

Kwon, O. C., Jung, K. H., You, H. C., \& Kim, H. E. (2009). Determination of key dimensions for a glove sizing system by analyzing the relationships between hand dimensions. Applied Ergonomics, 40(4), 762-766.

Lee, W. S., Yoon, S. H., \& You, H. C. (2010). Development of a 3D Semi-automatic Measurement Protocol for Hand Anthropometric Measurement. Proceedings of the Human Factors and Ergonomics Society, 54th Annual Meetings.

Li, Z., Chang, C. C., Dempsey, P. G., Ouyang, L., \& Duan, J. (2008). Validation of a three-dimensional hand scanning and dimension extraction method with dimension data. Ergonomics, 5l(1), 16721692.

Linghua, R., Xin, Z., Chuzhi, C., Taijie, L., \& Tingting, D. (2009). Anthropometric measurement of the hands of Chinese children. Lecture Notes in Computer Science, Digital Human Modeling, 5620, 46-54.

Ministry of Commerce, Industry and Energy. (2006). Development of hand measurement protocols for glove construction. Seoul: Goverment Printing Office.

Rosenblad-Wallin, E. (1987). An anthropometric study as the basis for sizing anatomically designed mittens. Applied Ergonomics, 18(4), 329-333.

Ryu, K. O., \& Suh, M. A. (2004). A study on the measurement of Korean women's hand-focusing on glove size. The Research Journal of the Costume Culture, 12(2), 262-278.

(Received 22 April 2016; 1st Revised 31 May 2016; 2nd Revised 5 July 2016; 3rd Revised 13 July 2016;

Accepted 15 July 2016) 